\title{
Impact of DME/TACAN on GNSS L5/E5a Receiver
}

\author{
Axel Garcia-Pena, Christophe Macabiau, ENAC \\ Olivier Julien, $u$-Blox AG, Switzeland \\ Mikael Mabilleau, Pierre Durel, GSA
}

\section{BIOGRAPHIES}

Axel GARCIA-PENA is a researcher/lecturer with the SIGnal processing and NAVigation (SIGNAV) research group of the TELECOM lab of ENAC (French Civil Aviation University), Toulouse, France. His research interests are GNSS navigation message demodulation, optimization and design, GNSS receiver design and GNSS satellite payload. He received his double engineer degree in 2006 in digital communications from SUPAERO and UPC, and his PhD in 2010 from the Department of Mathematics, Computer Science and Telecommunications of the INPT (Polytechnic National Institute of Toulouse), France.

Christophe MACABIAU graduated as electronics engineer in 1992 from the ENAC in Toulouse, France. Since 1994, he has been working on the application of satellite navigation techniques to civil aviation. He received his $\mathrm{Ph}$.D in 1997 and has been in charge of the signal processing lab of ENAC from 2000 to 2012. He is currently the head of the TELECOM team of ENAC.

Olivier Julien is a Senior Principal Engineer in u-blox AG, Switzerland since December 2018. He was the head of the Signal Processing and Navigation (SIGNAV) research group of the TELECOM laboratory of ENAC, in Toulouse, France. He received his engineer degree in 2001 in digital communications from ENAC and his PhD in 2005 from the Department of Geomatics Engineering of the University of Calgary, Canada. His research interests are turned towards the use of satellite-based navigation systems for safe navigation.

Mikael Mabilleau Mikael MABILLEAU is a standardisation engineer on SBAS in the EGNOS exploitation team of the European GNSS Agency (GSA). Since his graduation as engineer from the French civil aviation school (ENAC) in 2006, he has been involved in GNSS standardization activity carried by the main civil aviation standardisation bodies such as the EUROCAE WG 62 on Galileo, RTCA Special Committee 159 on GPS and the International Civil Aviation Organisation (ICAO) Navigation System Panel in charge of ICAO standard. Mikael is involved in the evolution of GNSS concepts (ARAIM and SBAS L1L5) supporting the development of the associated standards (SARPs and MOPS) for use in aviation

Pierre Durel graduated in 2002 from the University of Montpellier II as an engineer specialized in hyper-frequencies and optronics. $\mathrm{He}$ is working as EGNOS Services Engineer for the European GNSS Agency. He is involved in standardization activities dealing with DFMC SBAS for which he is secretary of EUROCAE Galileo Working Group 62.

\footnotetext{
ABSTRACT

GNSS L5/E5a interference environment is dominated by DME/TACAN and JTIDS/MIDS pulses causing a degradation of the effective C/N0 observed by the receiver. A time-domain blanker is implemented to mitigate their impact. RTCA DO-292 proposes a model to compute the $\mathrm{C} / \mathrm{N} 0$ degradation of the received useful signal by the increase of the noise PSD.

This paper focuses on the impact of DME/TACAN RFI signals. Simulated results as well as predicted results are presented for US and Europe scenarios. The predicted results are calculated from an updated C/N0 degradation formula with respect to RTCA DO292 proposed formula. The impact of GNSS receiver RFFE filter bandwidth and blanker threshold are evaluated.
} 


\section{1)INTRODUCTION}

Processing of GNSS received signals can be affected by received additive signals such as noise, multipath and interference. Radio Frequency Interference (RFI) sources are of various sorts and their nature and impact depends on the user application. In the context of civil aviation, it is important to identify and characterize the radio frequency interference relevant to the airborne GNSS receivers processing signals in the L1/E1 and L5/E5a bands. This characterization serves to determine the degradation of performance of these airborne GNSS receivers in L1/E1 and L5/E5a equipped with their relevant antenna which is then compared to operational performance thresholds, then serves to issue maximum tolerable levels of aggregate non aeronautical interference in order to protect the spectrum. Finally, this prediction of degradation of performance compared to functional thresholds is used to issue minimum requirements on these L1/E1 and L5/E5a antennas, as well as to issue minimum requirements to be imposed to airborne GNSS receivers operating at L1/E1 and L5/E5a bands. A long thread of activities led to the elaboration of various ICAO, RTCA and EUROCAE standards considering RFI. Currently, [RTCA, 2004] reflecting the relevant interference to L5/E5a is being updated to incorporate the evolutions of the RFI environment defined by DME/TACAN, JTIDS/MIDS, LDACS, SSR equipment and other GNSS systems operating at these bands, as well as the usage of this L5/E5a band for GALILEO E5a and SBAS L5/E5a datalink airborne signal processing. In addition, ICAO RFI mask of GNSS L5/E5a is now under definition and validation. These elements will then complement the current ICAO SARPs, draft EUROCAE and RTCA MOPS for GNSS L5/E5a airborne receivers.

In the course of the elaboration of the ICAO SARPs validation and of the update of [RTCA DO292, 2004], it has been proposed to revisit several elements of the worst-case link budget analysis in order to consolidate the overall link budget margin. This was deemed necessary since the link budget margin is expected to be very small. Among the axes of revision are:

- the analytical model representing the effect of the AGC/ADC and temporal blanker

- the DME/TACAN environment and its impact on a minimum operational/system performance requirements for a GNSS L5/E5a receiver

- the JTIDS/MIDS environment and its impact on a minimum operational/system performance requirements for a GNSS L5/E5a receiver

- The consideration of SSR and LDACS

This article specifically looks at the consolidation of the expected worst-case effect of the DME/TACAN environment on a GNSS airborne receiver.

The RFI impact on a GNSS receiver in civil aviation is usually modelled as the $\mathrm{C} / \mathrm{N}_{0}$ degradation observed at the receiver's correlator output, or equivalently, as an increase of the effective $\mathrm{N}_{0}$ denoted as $N_{0, e f f}$. Therefore, a decrease of the minimum available $\mathrm{C} / \mathrm{N}_{0}$, derived from the link budget and from the $N_{0, \text { eff }}$ calculation, implies a reduction of the $\mathrm{C} / \mathrm{N}_{0}$ margin between the minimum available $\mathrm{C} / \mathrm{N}_{0 \text {, eff }}$ and the different L5/E5a GNSS and SBAS L5 signal processing, acquisition, tracking, demodulation, $\mathrm{C} / \mathrm{N}_{0}$ threshold values. Concerning the revisit of several elements determining the $\mathrm{C} / \mathrm{N}_{0}$ margin, first, the model for the GNSS airborne receiver RF processing chain, namely the model for AGC/ADC and blanker is reviewed. In particular, the model for blanking function has gone under new scrutiny, with the prospect of the definition of a minimum blanker model. Second, the DME/TACAN environment is being reviewed. Models of impact of DME/TACAN on $\mathrm{C} / \mathrm{N}_{0}$ degradation are also revised. Next, the JTIDS/MIDS environment will be re-assessed, and the relevant models updated. LDACS and SSR will also need to be inspected.

Traditionally, the countermeasure adopted against pulse interference which is analyzed in civil aviation is the temporal domain pulse blanking method as described in [RTCA, 2004]. Temporal domain blanking method is easy to implement and computationally efficient. It can thus be considered as representative of what could be implemented in a minimum airborne receiver.

The expression of effective $N_{0}$ at the output affect temporal blanker is given below:

$$
N_{0, \text { eff }}=\frac{N_{0}}{1-b d c} *\left(1+\frac{I_{0, W B}}{N_{0}}+R_{I}\right)
$$

where $I_{0, W B}$ are all the wideband (non-pulsed) continuous RFI contributions (usually the other GNSS signals falling in the L5/E5a band). Setting the blanking threshold correctly can be challenging due to the trade-off between the Blanking Duty Cycle, abbreviated as $b d c$ (percentage of samples set to zero by the blanker) also sometimes called the Pulse Duty Cycle of the blanker abbreviated as $P D C$ depending on the authors [RTCA, 2004], and the $R_{i}$ (the below-blanker interfering-signal-to-thermal-noise ratio) parameters since both of them directly impact the effective noise, $N_{0}$, of the received signal after blanking. On one hand, a low threshold removes 
the majority of the signal samples containing interference because they will exceed the threshold (reduction of $R_{I}$ ), but such a low threshold causes that fact a higher percentage of time the noise alone is enough to trigger the zero-setting process causing a "false alarm" (increase of $b d c$ ), and therefore this setting of samples to 0 due to noise and interference also suppresses the useful GNSS signal energy. On the other hand, a high threshold value decreases the "false alarm" events due to noise and lets some interference and useful GNSS signal energy go through (decrease of $b d c$ ), but also does not appropriately suppress the interference samples (increase of $R_{I}$ ). In the situation of pulsed interference, proper blanker threshold selection is thus the result of a compromise and a crucial factor of performance in such blanking methods.

The general aim of this paper is thus to present ways to predict the $N_{0, \text { eff }}$ and thus to compute $b d c_{D M E / T A C A N}$ (also termed $\left.P D C_{D M E / T A C A N}\right)$ and $R_{I, D M E / T A C A N}$ using the general methodology to assess the effect of the different interference sources on the increase of the background noise for Galileo E5a or GPS L5 signals in presence of DME/TACAN pulses only as interference sources. The specific objectives of this paper can be listed as follow:

1. Present the model for DME/TACAN signals

2. Show a brief graphical analysis of 2018 US and Europe DME/TACAN databases

3. Introduce a model for DME/TACAN degradation including analysis of collisions

4. Present simulation results as a function of receiver bandwidth and blanking threshold

5. Focus the analysis on US and Europe DME/TACAN Hot Spots

6. Document findings and draw new conclusions on the effect of DME/TACAN which can be further recommended in ICAO SARPs and EUROCAE/RTCA MOPS development

\section{2)MODEL FOR DME/TACAN SIGNALS}

DME, and its military equivalent, TACAN, are two systems used by aircraft to know their distance to a ground station, which position is known. The systems operate as follows: the aircraft DME equipment (called interrogator) sends pulses to ground stations. Once the interrogation is detected, the station transponder replies to the interrogator. The distance is then determined by the aircraft interrogator by measuring the time elapsed between each pulse transmitted by the interrogator and the reception of its corresponding reply pulse from the transponder. This time corresponds to twice the distance between the aircraft and the station, plus fixed processing time inside the ground station. The characteristics of these systems, as well as their impact on GNSS L5 is presented in [Bastide, 2004].

Based on the propagation delay, the aircraft interrogator equipment calculates the (slant range) distance from the transponder to its current location. The round trip time is usually much less than the interval between transmitted pulse pairs; hence the interrogator will receive a response before it sends out the next pulse pair. A DME ground-based transponder serves all (recommended maximum number of 100 aircrafts) aircrafts within a designated radius of coverage. During the search phase, the aircraft interrogator sends, on average, between 40 and 150 pulse pairs per second (ppps) continually. Regarding the tracking phase, the pulse repetition frequency (PRF) is much lower because the search is required to be completed quickly and the frequency is between 10 and 30 pulse pairs on average. Modern DME interrogators currently use digital correlation techniques which mean that they can interrogate at much lower rate: 30 ppps during the search and 5 ppps for tracking.

The automatic standby system prevents the airborne interrogator to transmit a signal when out of range of a DME beacon. Its aim is to reduce the energy waste and to increase the equipment longevity. It relies on the detection of minimum signal strength or minimum pulse rate. Given this airborne receiver function, a specific DME ground beacon must always transmit a minimum number of pulses otherwise airborne interrogators would never send any interrogation even when in the beacon range. If there are enough aircrafts interrogating the beacon then there is no issue since the beacon would transmit enough pulse pairs further detected by an airborne DME interrogator entering the radius of coverage. In the case where no interrogations are received the ground beacon is designed to transmit pulsed pairs called squitter to distinguish them from replies. Thus a ground beacon is specified to transmit at least 700 pulse pairs per second apportioned between distance replies as well as squitter. This minimum rate is maintained by varying the ground beacon receiver sensibility. When no interrogations are received, sensitivity is set sufficiently high for noise to trigger the transmitter. When interrogations begin to be received and processed, the sensitivity is decreased to not exceed a given maximum rate.

Some reflected or echo interrogations may arrive to the ground beacon a short time after the direct interrogation depending on the terrain configuration. The beacon must be prevented from triggering the transmitter after reception of such a signal to avoid the aircraft receiver tracking an echo. The way employed to guarantee this protection is to suppress the receiver function of the ground beacon for a deadtime period of up to $60 \mu \mathrm{s}$. The DME/TACAN system operates in four modes (X, Y, W and Z) and only the Xmode replies in the 1151-1213 MHz frequency band that overlaps the E5a/L5 and E5b bands. 
According to [RTCA, 2004], only the signals emitted in the band of interest of the study disturb GNSS receivers operations. Indeed, the band of interest is the E5a/L5 one and equals [1164 MHz; $1191 \mathrm{MHz}$, The aircraft's DME interrogators emitting their signals between 1025 and $1151 \mathrm{MHz}$ or between $1191 \mathrm{MHz}$ and $1215 \mathrm{MHz}$, they are ignored herein.

The main on-board signal representing a risk for E5a/L5 is transmitted by the DME interrogator. Indeed the nearest airborne channel is $26.45 \mathrm{MHz}$ below E5a/L5. Although the produced pulses are highly spectrally constrained and attenuated at this central frequency by the RF Front-End, they could produce saturation at E5a/L5 stretching the pulses duration, but their impact is constrained to $b d c_{D M E / T A C A N \_ \text {airborne }}=0.0026$ and $R_{I, D M E / T A C A N_{-} \text {airborne }}=0.002$. Interrogators from nearby aircraft were shown to induce a negligible impact [RTCA, 2004].

The study therefore focuses on DME ground stations, as they emit their signals between 962 and $1213 \mathrm{MHz}$, which includes the above defined band of interest. The only ground DME beacons of interest in this analysis are the beacons operating in X mode, for which the central frequency is larger or equal to $1151 \mathrm{MHz}$.

The emitted signal of an individual DME station is composed of a pair of Gaussian pulses modulated by a cosine, which can be modelled as:

$$
s(t)=\sqrt{P} \sum_{n=1}^{K}\left(e^{-\frac{\alpha\left(t-t_{k}\right)^{2}}{2}}+e^{-\frac{\alpha\left(t-t_{k}-\Delta t\right)^{2}}{2}}\right) \cos \left(2 \pi f_{i} t+\theta_{i}\right)
$$

Where:

- $\quad P$ is the interference beacon transmitting peak power (dBW),

- $f_{i}$ is the carrier frequency of the DME/TACAN signal $(\mathrm{Hz})$,

- $\alpha=4.5 \cdot 10^{-11} \mathrm{~s}^{-2}$

- $\Delta t=12 \mu \mathrm{s}$ is the inter pulse time separation,

- $t_{k}$ is the emission time of the kth pulse pair

- $\theta_{i}$ is the DME/TACAN signal initial carrier phase shift.

Figure 1 (left part) represents a normalized DME/TACAN pulse pair, modulated at $14 \mathrm{MHz}$. Figure 1 (right part) represent the normalized pulse complex envelope of a DME/TACAN signal.
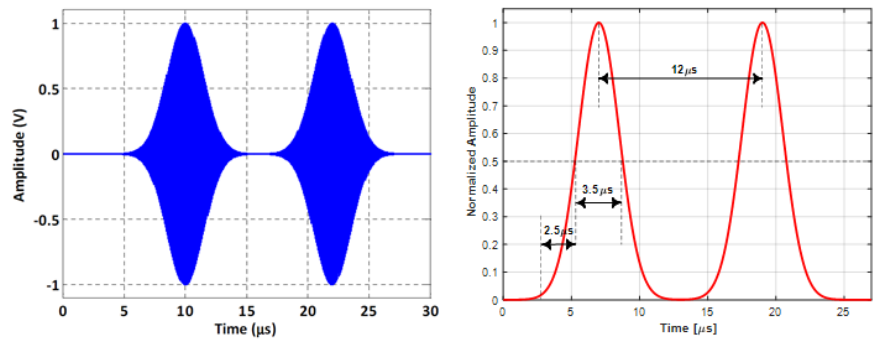

Figure 1: DME signal pattern

These pulsed signals will then propagate to the aircraft receiver antenna and will then superimpose to thermal noise and to the other received signals, nominally from the useful GNSS satellite, and from the other GNSS satellites.

Various pulsed interference blanking methods have been previously studied ranging from frequency notch filtering, temporal domain blanking and temporal-frequency hybrid filtering [Hegarty et al., 2000], [Grabowski et al., 2002], [Gao et al., 2013], [Raimondi, 2008], [Shallberg et al., 2018]. The receiver considered in our study includes a temporal blanker as described in paper [Julien et al., 2019]. This temporal blanker is typically modeled as a detector making a decision on zeroing ADC samples based on the comparison of an estimated sampled signal power over a $1 \mu \mathrm{s}$ window to a blanking threshold. As shown in [Julien et al., 2019], the optimum blanking threshold selected by the Rx designers can vary by 1 or $2 \mathrm{dBs}$ around the optimal value of $B_{T H}=-120 \mathrm{dBW}$ or $B_{T H}=-$ $121 \mathrm{dBW}$. It was shown that $B_{T H}=-121 \mathrm{dBW}$ is better suited to cope with JTIDS/MIDS scenarios such as original Case VIII scenario.

To obtain the results given in this current paper, we considered the ideal application of DO292 blanker in such a way that we determined from the theoretical envelope of each of the received DME/TACAN signals above the samples which are blanked due to 
the specific current pulse considered, and then the final blanking function was built as the logical AND combination of each individual DME/TACAN blanking vector.

\section{B) US hot spot scenario defined in DO292}

DO292 defines the US DME/TACAN hot spot environment at FL400 characterized at the time of DO292 writing in DO292 table E8 by specifying beacons locations, frequency, and received power for a total of 39 beacons.

DO 292 actually identifies 3 US hot spots from the theoretical $C / N_{0}$ degradations (equivalently $N_{0, \text { eff }}$ degradation) due to RFI DME/TACAN signals computed using this DO292 table E-8 DME/TACAN database. Figure 2 shows the US map with the theoretical degradation results which were calculated using RTCA DO292 formulas (5) and (8).

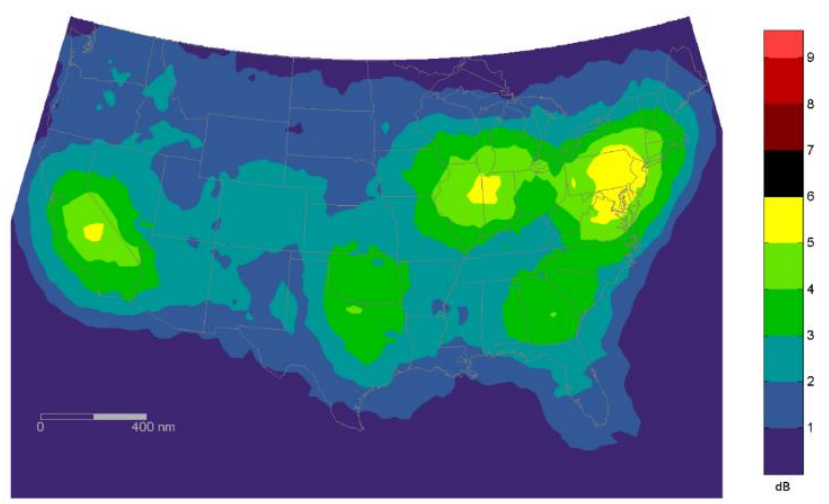

Figure 2: DME/TACAN beacon $N_{0, e f f}$ degradation factor contours at FL400 shown in DO292 [RTCA, 2004]

In this analysis, out of the three identified hot spots, the worst one is selected: near Harrisburg, PA, at $40^{\circ}$ Nord latitude and $76^{\circ}$ West longitude.

\section{B) US hot spot scenario from 2018 database}

The database for DME/TACAN characteristics over continental US except Alaska as of sep 2018 contains DME/TACAN positions as shown below in Figure 3.

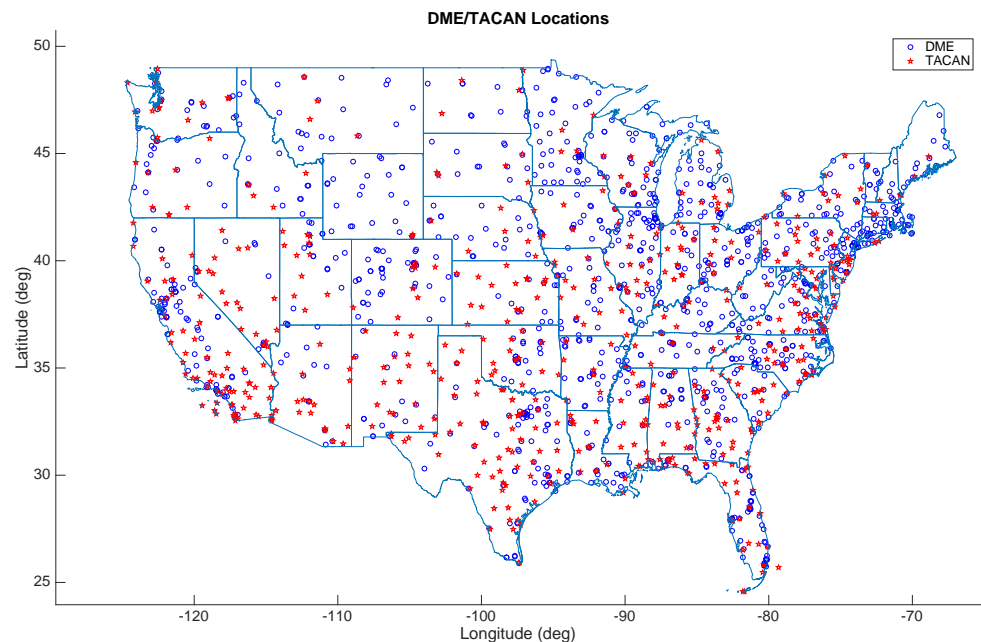

Figure 3 : Location of US DMEs and TACANs in 2018 database used for $\mathrm{C} / \mathrm{N}_{0}$ degradation prediction.

The 2018 database for DME/TACAN characteristics over the US illustrated in Figure 3 is processed to determine which DME/TACAN ground beacons are visible by aircraft receivers that have a longitude in the range $-169 \ldots-65$ degrees and a latitude in the range $25 \ldots 72$ degrees when we include Alaska in the analysis. The visibility is declared when the straight-line distance between the aircraft and the beacon is within the radio horizon computed with an Earth Radius multiplied by 4/3. 
Then, the power level of the signal received from each visible ground DME/TACAN beacons is computed. This starts by reading the peak power at the beacon equipment output in the US database, retrieving $2 \mathrm{~dB}$ to account for cable/connector losses before the antenna, and adding $8 \mathrm{~dB}$ to reflect the DME/TACAN antenna gain in the direction of maximum gain, which is equivalent to adding $6 \mathrm{~dB}$ to the peak power read in the US database. We then obtain the Equivalent Isotropic Radiated Power in the direction of maximum beacon gain to which we add a model of the normalized beacon gain (peak value $=0$ ) of the DME or TACAN antenna pattern. We then compute the free space losses at the specific DME or TACAN carrier frequency from the value of the straight-line distance between Tx and Rx. Finally, we add the gain of the aircraft GNSS Rx antenna usually at negative elevation angles.

The processing of the 2018 US DME/TACAN database shows that the largest C/N0 degradation for an aircraft at FL400 is observed when the aircraft is located at latitude 40 deg North and longitude 76 deg West. At this point in the sky, a total of 275 active DME/TACAN stations are visible, among which 92 DME/TACAN ground beacons have an operating frequency larger or equal than $1151 \mathrm{MHz}$ therefore operating in X mode as considered in our simulations. Among these 92 beacons, it is predicted that $14 \mathrm{DME}$ stations induce a received signal level above the blanking threshold $\mathrm{Bth}=-121 \mathrm{dBW}$ and 21 TACAN stations induce a received signal level above the blanking threshold $\mathrm{Bth}=-121 \mathrm{dBW}$.

The number of DME/TACAN in the $1166 \mathrm{MHz}-1187 \mathrm{MHz}$ bandwidth is 33 . Figure 4 shows the received power level distribution of each DME/TACAN in the 1151-1213MHz bandwidth as a function of their frequency and blanking threshold Bth=-121 dBW.

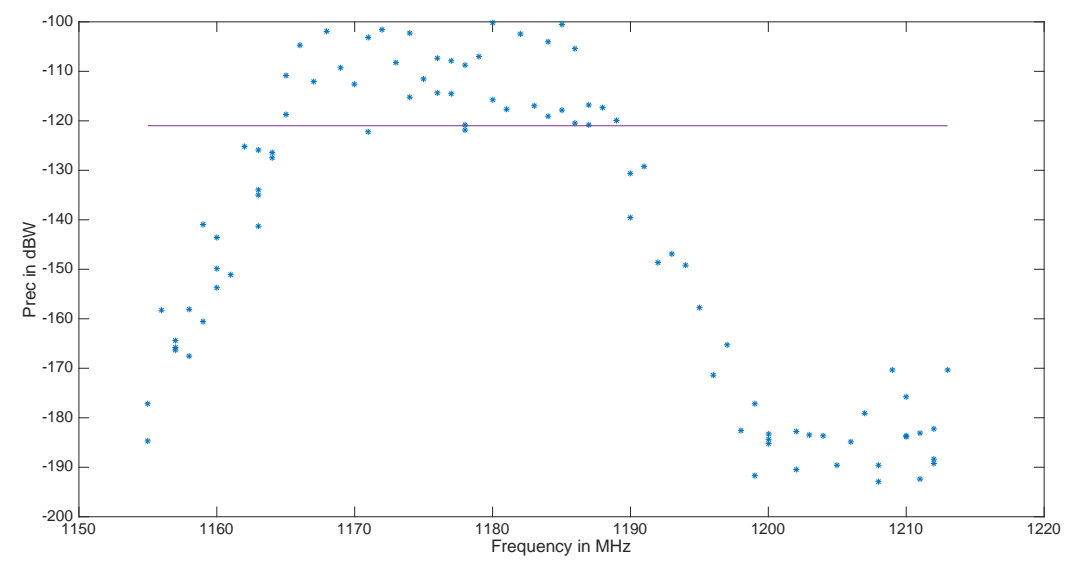

Figure 4 : Predicted received power level at FL400 over the US hot spot from the 92 DME/TACAN in the 2018 US DME/TACAN database in the 1151-1213MHz bandwidth (33 stations in $1166 \mathrm{MHz}-1187 \mathrm{MHz}, 35$ stations above $-121 \mathrm{dBW}$ blanking threshold).

\section{C) Europe hot spot scenario from 2018 database}

The database for DME/TACAN characteristics over Europe as of sep 2018 contains DME/TACAN positions as shown below.

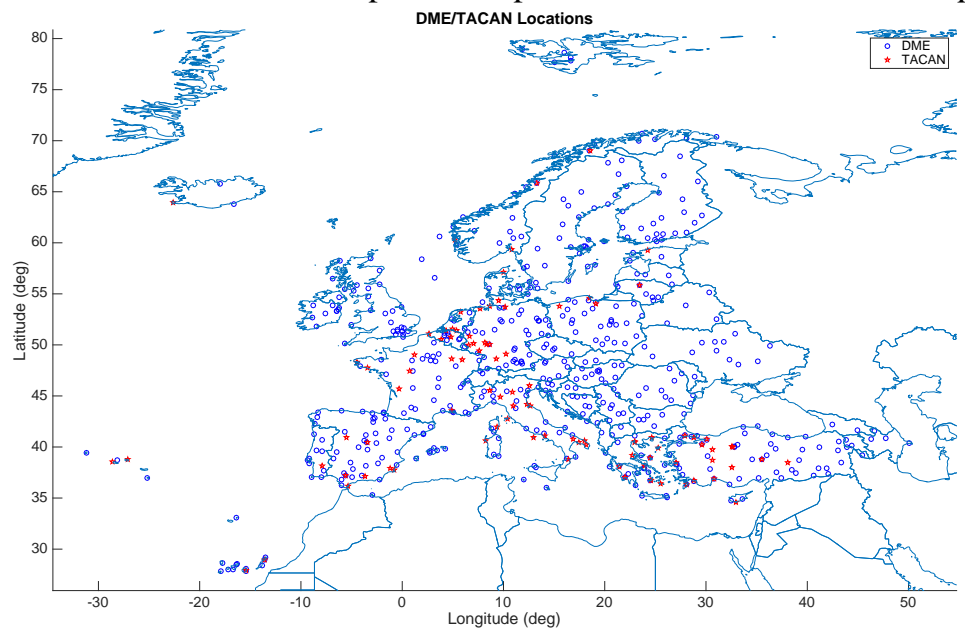

Figure 5: DME/TACAN beacons in Europe

The hot spot for GNSS L5 C/N0 degradation due to DME/TACAN for an aircraft at altitude FL400 is located at latitude 50.5 deg North and longitude 5.5 deg East. 
At this point in the sky, a total of 93 active DME/TACAN ground beacons are visible and have an operating frequency larger or equal than $1151 \mathrm{MHz}$ therefore operating in X mode as considered in our simulations. Among these 93 beacons, it is predicted that 26 DME stations induce a received signal level above the blanking threshold Bth=-121 dBW and 11 TACAN stations induce a received signal level above the blanking threshold $\mathrm{Bth}=-121 \mathrm{dBW}$.

The number of DME/TACAN which are in the $1166 \mathrm{MHz}-1187 \mathrm{MHz}$ bandwidth is 39 . The next figure shows the distribution of the received power level of each DME/TACAN in the 1151-1213MHz bandwidth as a function of their frequency as well as the blanking threshold Bth=-121 dBW.

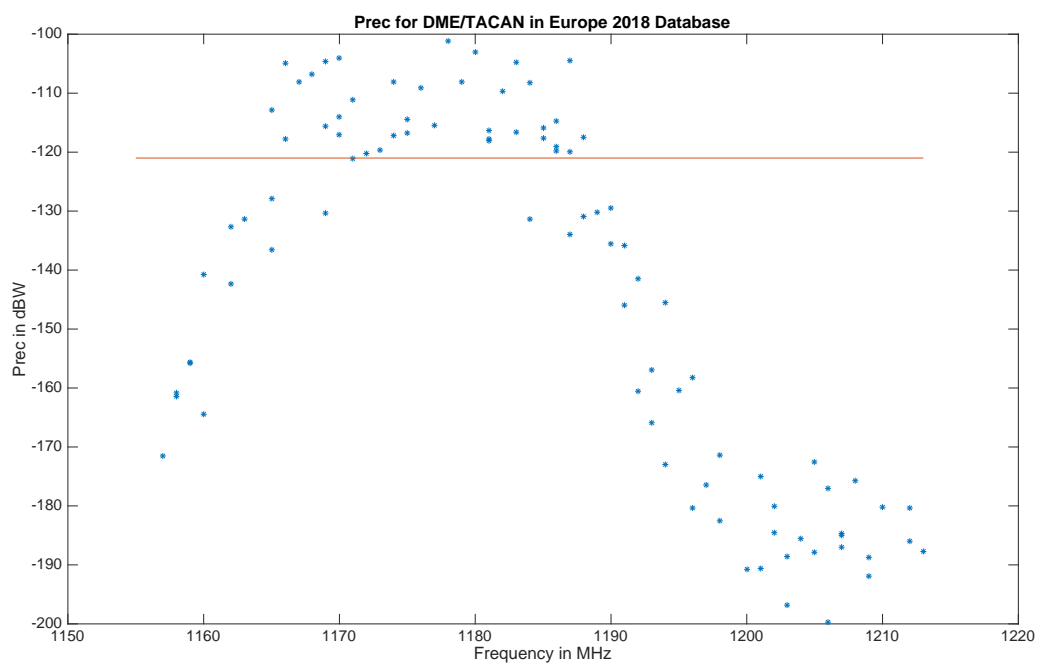

Figure 6: Predicted received power level at FL400 over the European hot spot from the 39 DME/TACAN in the 2018 European database in the 1151-1213MHz bandwidth ( 39 stations in $1166 \mathrm{MHz}-1187 \mathrm{MHz}, 37$ stations above $-121 \mathrm{dBW}$ blanking threshold).

\section{3) MODEL FOR DME/TACAN DEGRADATION}

\section{A) General analytical model}

In order to understand the DO-292 degradation analytical model, a generic civil aviation GNSS receiver structure as well as the behaviour and effect of its components on the received signals are described. In Figure 7, the receiver structure is presented.

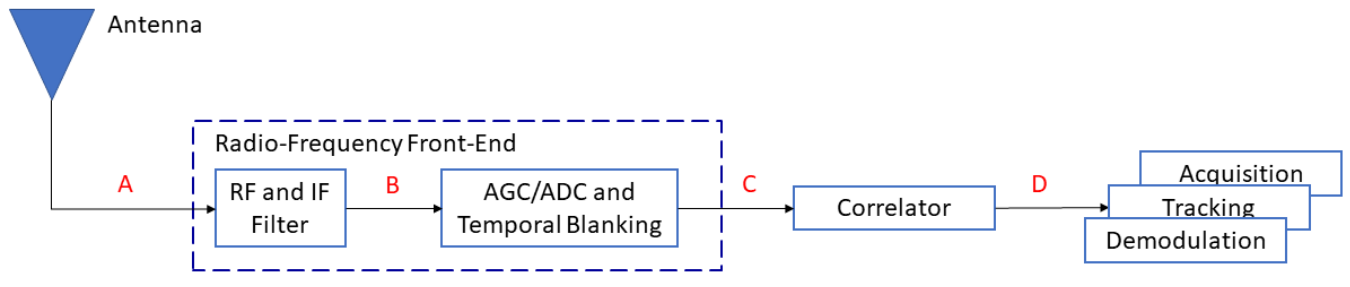

Figure 7: Generic civil aviation GNSS receiver block scheme

First, the antenna is the element responsible of capturing the incoming signal: at the antenna port (point A), there is a mix of all incoming signals; useful signals, GNSS and SBAS signals, and RFI signals such as DME/TACAN, JTIDS/MIDS, etc.

The effective $\frac{C}{N_{0}}$ is the ratio of RF useful GNSS signal power to equivalent noise density level as if all subsequent elements (RFFE, AGC/ADC, correlator) are ideal and do not bring losses and as if no interference (and no blanker) is present but only white noise. The model of the effective $\frac{C}{N_{0}}$ at point A is presented in [Garcia-Pena et al., 2020] and is such that:

$$
\left(\frac{C}{N_{0}}\right)_{e f f}=\frac{P_{u} L_{u}(1-b d c)^{2}}{\left(I_{0,0}+\sum_{l=1}^{L} I_{0, l}\right)}=\frac{P_{u} L_{i m p, u}(1-b d c)^{2}}{\left(I_{0,0}+\sum_{l=1}^{L} I_{0, l}\right) / \beta_{0}}
$$




$$
\beta_{0}=\int_{-\infty}^{+\infty}\left|H_{R F}(f)\right|^{2} \bar{S}_{c_{m}}(f) d f
$$

where

- $\quad P_{u}$ is the received useful signal power at the antenna input. We note $C=P_{u} L_{i m p, u}$

- $\quad b d c$ is the blanker duty cycle if a blanker is used

- $\quad L_{u}$ corresponds to losses on the useful signal due to the RF front-end filter, quantization, correlation, etc.

- $I_{0, l}$ is the equivalent noise PSD level created by each individual interference determined upon knowledge of the power of their corresponding correlator output

- $I_{0,0}=N_{0} \beta_{0}(1-b d c)$ is the equivalent thermal noise PSD level at the RFFE input

- $\quad \beta_{0}$ is the noise power degradation due to RFFE filter and correlator

- $\bar{S}_{c_{m}}$ is the normalized PSD of the local replica of the $m^{\text {th }}$ PRN code used by the correlator

- $H_{R F}(f)$ is the baseband transfer function of the equivalent RFFE plus antenna filter

Note that ahead of point A the useful GNSS signal is sensed by the receiver antenna with an antenna gain leading to the observed useful GNSS signal pwer $P_{u}$ that may be different from the antenna gain offered in the direction of the interfering signals.

Once the signals have been captured by the antenna, they are passed to the Radio-Frequency Front End (RFFE) block. This block amplifies the received signals, shifts them from their received signal frequency carrier to the intermediate frequency and filters them. The filtered signals are modelled in Figure 7 at the RF (Radio-Frequency) and IF (Intermediate Frequency) filters output at point B. RTCA DO292 defines the joint effect of these two filters plus the antenna filtering effect with an equivalent filter such that for this $20 \mathrm{MHz}$ filter, the passband ends at $10 \mathrm{MHz}$ away from central frequency and the stopband starts at $22.7 \mathrm{MHz}$ away from the central frequency, creating a transition band with slope $5.5 \mathrm{~dB} / \mathrm{MHz}$.

The RFFE block is also responsible for digitizing the filtered signals with the application of the ADC (Analog-Digital-Converter) coupled with the AGC (Automatic Gain Control). In a civil aviation receiver the pulse blanker is introduced at that point. As explained in the introduction, the pulse blanker is a device which blanks (sets to 0s) the time and/or frequency samples of the incoming signal (mix of signals) upon appropriate detection of the presence of a pulse. The assumed AGC/ADC - blanker mechanism is described in section 2.5.4 of RTCA DO292. It is assumed to comprise a first ADC stage, then the digitized and blanked signal is found at point C. For optimal operation, the estimated signal power used to drive the AGC should be observed at the output of the ADC which is following the pulse blanker in order to ensure that high-power samples are not saturating the AGC/ADC and that the blanked signal spans the final ADC quantization range. For simplification purposes, in this paper, the effect of the AGC/ADC and the effect of its coupling with the blanker are not considered.

In DO292, a version of the defined blanker is a temporal blanker called instantaneous blanker. This blanking method removes all the incoming signal time samples which have an instantaneous power over a given threshold. The main limitation of the instantaneous blanking method proposed in DO292 is that the performance obtained using its definition does not correspond with the analysed performance in some parts of the document. In fact, the performance presented in DO292 does not consider that RFI signals (DME/TACAN, JTIDS/MIDS, etc) are modulated around a carrier frequency. This means that the DO292 analysed performance assumed an instantaneous blanking method over the incoming signal envelope. Efficient blanking mechanisms were evaluated in [2].

Finally, digitized and blanked signals are fed to the correlator and it is at its output (point D) where the impact of the RFI signals and the blanking method is measured. The chosen figure of merit is the signal $C / N_{0}$; or more specifically, the difference between the $C / N_{0}$ when only the useful signal is present at the receiver antenna port (no RFI signals) and the $C / N_{0}$ when the useful signal and RFI signals are present at the receiver antenna port, also called effective $C / N_{0}$ or $C / N_{0, e f f}$. The difference between these two $C / N_{0}$ values is also called the degradation introduced by the RFI signals.

Although the blanking method is going to affect the power of the useful signal (part of the signal is removed and this its power is decreased), DO292 proposes to model the $C / N_{0, e f f}$ by defining an equivalent $N_{0, e f f}$. Note that $N_{0, e f f}$ represent the effective noise power spectrum density that a receiver will observe at the correlator output if the receiver captures a useful signal with power $C$ at the correlator output; and no RFI signals are present. In other words, DO292 proposes a generic formula to compute the degradation of the $C / N_{0}$ through the increase of the background noise due to pulsed and continuous RFI. 
In order to mathematically model $N_{0, e f f}$, the following considerations about the blanking and the incoming signals must be considered. First, although all received GNSS and SBAS signals are by definition useful signals, since the receiver has to isolate them one-by-one to exploit them, they are also considered RFI signals: if the receiver is trying to isolate GNSS (or SBAS) signal $i$ in one correlator block, all the other GNSS (or SBAS) signals $j, j \neq i$, falling in the L5/E5a band are considered RFI signals. These signals are wideband (non-pulsed) signals and its contribution is modelled with term $I_{0, W B}$. Note that the blanking method will not target these signals since they are continuous and thus, the blanking method settings will be determined by the pulsed RFI signals, such DME/TACAN and JTIDS/MIDS.

Second, pulsed RFI signals impacts in two different ways $N_{0, e f f}$ :

- Part of the signal is removed due to the blanking and since the impact on the removed useful signal power, $(1-b d c)^{2}$, is higher than the impact on the noise power, $(1-b d c)$, the equivalent $N_{0, \text { eff }}$ can be seen to be increase by a factor of $1 /(1-$ $b d c) . b d c$ is the blanker duty cycle, or in other words, the percentage of time the incoming signal is blanked $(b d c \in[0,1])$.

- Not all the RFI signal samples have a power above the threshold; thus, there is a part of the RFI signal that is not removed and its influence must be added to the thermal noise; $R_{I}$ is the below-threshold interfering-signal-to-thermal-noise ratio

The mathematical model of $N_{0, e f f}$ is given below:

$$
N_{0, e f f}=\frac{N_{0}}{1-b d c} *\left(1+\frac{I_{0, W B}}{N_{0}}+R_{I}\right)
$$

Where:

$$
\begin{gathered}
R_{I}=\sum_{i=1}^{I} R_{I, i} \\
b d c=1-\prod_{i=1}^{I}\left(1-b d c_{i}\right)
\end{gathered}
$$

Where:

- $\quad I$ is the total number of RFI signals

- $\quad R_{I, i}$ is the source $i$ below-blanker interfering-signal-to-thermal-noise ratio

- $\quad b d c_{i}$ is the $b d c$ of interfering source $i$

Finally, from the previous explanations and equations (5), (6) and (7), it can be observed that the settings of the time domain blanking threshold are very important and will determine the final $N_{0, e f f}$, and thus the degradation caused by the RFI signals. On one hand, a low threshold removes the majority of the signal samples containing interference (reduction of $R_{I}$ ) but a higher percentage of time the noise alone is enough to trigger the zero-setting process causing a "false alarm" (increase of $b d c$ ). On the other hand, a high threshold value decreases the "false alarm" events (decrease of bdc) but also does not appropriately suppresses the interference term (increase of $R_{I}$ ). Proper threshold selection is thus a crucial factor of performance in such blanking methods.

\section{B) Computation of $\boldsymbol{R}_{I}$}

Since $R_{I}$ represents the contribution of the below-blanker interfering-signal-to-thermal-noise ratio, its computation depends on the mathematical model of the RFI signal at the blanker output. More specifically, it depends on the power spectrum density (PSD) of such signal and note that, due to the blanking method, the signal time structure as well as its PSD can be quite different from the ones of the signal before-blanking. For example, DO292 proposes a formulation that assumes that the blanked interfering signals are completely uniformly spread over the receiver bandwidth. The formula is given below for pulse interference $i, R_{I, i}$ :

$$
R_{I, i}=\beta_{i} \frac{P_{P e a k, i} \times d c_{i}^{p b}}{N_{0} \times B W}
$$

Where:

- $\quad P_{\text {peak }, i}$ is the peak power of the interfering source $i$ at the antenna output 
- $\quad d c_{i}^{p b}$ is the post-blanker duty cycle of pulsed interference source $i$ that represents the part of the signal power that goes through the blanker so that $P_{P e a k, i}(1-b d c) d c_{i}$ is the post-blanker interfering source signal power

- $B W$ is bandwidth of the equivalent transfer function of the RFFE block plus antenna

- $\quad N_{0}$ is the noise power spectrum density

- $\quad \beta_{i}$ is the RFFE filter frequency dependent rejection (FDR) to the received RFI signal

- $\bar{S}_{l, B}(f)$ is the baseband normalized $l^{\text {th }}$ interfering signal PSD

- $\Delta f$ is approximated as the frequency difference between the the $l^{l h}$ interfering signal central frequency and the receiver central frequency, in this case the L5 frequency

$$
\beta_{i}(\Delta f)=\int_{-\infty}^{+\infty}\left|H_{R F}(f)\right|^{2} \bar{S}_{i, B B}(f-\Delta f) d f
$$

However, simulation results have shown that the interfering signal PSD after the blanking is not in fact uniformly spread and thus, the hypothesis considered in DO292 appears to be too conservative. We propose to use for DME/TACAN signals the following formula which takes into account the true PSD of the signal entering the correlators [Garcia-Pena et al., 2020]:

$$
\begin{gathered}
R_{I, i} \sim \frac{1}{\beta_{0} N_{O}(1-b d c)} \sum_{i=1}^{N}\left(\beta_{i}\left(\Delta f_{i}\right) P_{p e a k, i} d c_{i}\left(1-l d c_{i}\right) \operatorname{SSC}_{i}^{p b}\left(\Delta f_{i}\right)\right) \\
\operatorname{SSC}_{i}^{p b}(\Delta f)=\int_{-\infty}^{+\infty} \bar{S}_{i, P B f}(f-\Delta f) \bar{S}_{c_{m}}(f) d f \\
\beta_{l}(\Delta f)=\int_{-\infty}^{+\infty}\left|H_{R F}(f)\right|^{2} \bar{S}_{i, B B}(f-\Delta f) d f
\end{gathered}
$$

Where:

- $\quad P_{\text {peak }, l}$ is the peak power of the $l^{\text {th }}$ interfering source at the antenna output $H_{R F}(f)$ is the equivalent transfer function of the RFFE block plus antenna

- $\quad \bar{S}_{l, B B}(f)$ is the $l^{\text {th }}$ baseband before-blanker normalized interfering signal PSD

- $\bar{S}_{l, P B f}(f)$ is the $l^{\text {th }}$ baseband filtered post-blanker normalized interfering signal PSD. Note that this PSD represents the PSD of the incoming interfering signal, $S_{B B, i}$, filtered by equivalent transfer function of the RFFE block plus antenna, $H_{R F}(f)$, which is then blanked (either because the interfering signal itself triggers the blanker or or because or interfering signals do)

- $\quad d c_{i}$ is the duty cycle of pulsed interference source $i$ when no blanker is present

- $l d c_{i}$ is the $i^{t h}$ interfering signal duty cycle loss due to blanking; $l d c_{i} \epsilon[0,1], d c_{i}^{p b}=d c_{i}\left(1-l d c_{i}\right)$

The proposed expression is a more accurate representation than the formula defined by DO292 but it requires a model for $S_{P B, i}$ for each source that is essentially bdc-dependent.

This formula (10) assumes the knowledge of the PSD of the interfering signals at the correlator input. This assumption is debatable in the context of a receiver that uses a blanker since the received interference might be partially blanked depending upon the other interference sources received at the same time. As a consequence, the interference PSD at the correlator input cannot, a priori, be considered as the interference PSD at the output of the RF front-end filter. As the blanking process sets to 0 some input signal samples upon blanking, this changes the frequency components distribution and in particular adds some high frequency components that may change and potentially increase the cross-correlation between the locally generated code and the blanked signal. Therefore, it is more appropriate to use the post-blanked interference spectrum rather than the input interference spectrum.

These models also assume a temporal blanker implementation in the GNSS receiver. Temporal blanker analysis were presented in [2] and the most appropriate model has been shown to be the sliding window average power blanker where the blanker criterion is an average of square magnitude of samples within a time window (i.e. $0.5 \mu \mathrm{s}$ or $1 \mu \mathrm{s}$ ) and this average power is not purely the peak 
power envelope at each epoch. The results presented in this current paper were obtained with the ideal DO-292 blanker which is blanking the pulsed interference signals by comparing the mathematical magnitude of the theoretical expression of the complex envelope of each pulse to the blanking threshold, the final blanking mask being equal to the OR of each individual pulse blanking.

\section{4)SIMULATION RESULTS}

\section{A) Proposed Computation of $R_{I, D M E / T A C A N}$}

It is to be noted that the shape of post-blanker DME/TACAN PSD will be mainly affected by 2 elements:

- The bdc: the blanking rate will influence how the pulses are blanked

- The magnitude of the DME/TACAN signal:

$\circ$ if the signal has a high amplitude and triggers the blanker, the post-blanker residual signal will have a specific shape since the "center of each pulse" will be blanked

- in particular, when the bdc starts getting small, the effect of the blanker triggered by the DME/TACAN signal itself becomes dominant over the effect of triggering of the blanker by "external events (other DME/TACAN)

$\bigcirc \quad$ if the signal has a low amplitude and does not trigger the blanker (by itself), the post-blanker signal is only affected by "external" events triggering the blanker

To do that, simulations were run at the so-called DME/TACAN US hot spot using the DME/TACAN received signal characteristics provided by Table E-8 of [1]. The simulation process to model the post-blanked DME/TACAN PSD was as followed:

- Simulate the DME/TACAN environment

- Record the blanking instants assuming the blanking methodology used in [1]

- Apply the blanking instant on a single DME or TACAN signal

- Compute the resulting PSD over a given period of time

This resulted in the modeling of the normalized post-blanking PSD of the DME/TACAN signals according to Figure 8. At the hot spot, the blanker duty cycle is above 60\% (as it will be seen in a later section). It can be seen that the high level of blanking significantly spreads the normalized DME/TACAN PSD. At this high bdc, no major differences were observed between high and low amplitude DME/TACAN signal. However, it is not flat and maintains a majority of its power at each transmission frequency: the PSD is more than $10 \mathrm{~dB}$ below its peak value after $2 \mathrm{MHz}$. Compared to the model taken by [1], this will result in a higher $R_{I, k}$ when the DME/TACAN is at a frequency close to the L5 frequency, and a lower $R_{I, k}$ when it is at a frequency away from the L5 frequency.

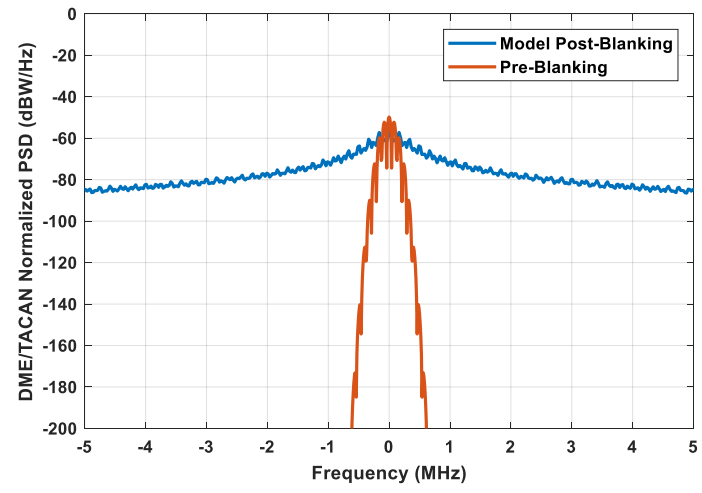

Figure 8 - Representation of the Original DME/TACAN normalized PSD and the Proposed Post-Blanking Model

\section{B) Simulation results at DO-292 Table E-8 US Hot Spot}

We first computed the predicted GNSS L5 C/N0 degradation results observed by an aircraft al FL400 at the US hot spot using the database in DO292 Table E-8, with a temporal blanker blanking threshold of Bth=-120dBW. This is done in order to compare results with other existing results.

As results of predicted C/N0 degradation were computed at the time of RTCA DO292 written and reported in DO292 table 10-3, this situation is taken in this paper as a reference situation to evaluate our prediction formula (10). For that, we will compare our C/N0 
degradation results with $\mathrm{C} / \mathrm{N} 0$ degradation results given in DO292 table 10-3 corresponding to DME/TACAN environment table E8. But as our formula has been designed to try and better reflect reality and stick closer to observed degradation, it is anticipated that there will be differences between our prediction of C/N0 degradations from formula (10) compared to RTCA DO 292 formula (8). Therefore, to bring validation of our formula (10), we also simulated the reception of DME/TACAN signals from DO292 Table E-8 environment by generating these DME/TACAN signals and injecting them on top of GNSS useful signal and thermal noise into a $\mathrm{Rx}$ simulator.

Considering this environment from DO292 table E-8 with the DME/TACAN received power given in table E-8, the degradation results when injecting these simulated DME/TACAN signals into our Rx simulator are shown in Table 1 when setting the temporal blanker threshold to $B_{T H}=-120 \mathrm{dBW}$.

\begin{tabular}{|l|l|l|}
\hline$B_{T H}=-120 \mathrm{dBW}$ (DO292 blanking) & $20 \mathrm{MHz} \mathrm{Rx}$ & $12 \mathrm{MHz} \mathrm{Rx}$ \\
\hline $\mathrm{bdc}$ & 0.6195 & 0.4652 \\
\hline Estimated $R_{I}$ & 0.3783 & 0.5107 \\
\hline Degradation $(\mathrm{dB})$ & 5.59 & 4.51 \\
\hline
\end{tabular}

Table 1 : Results of observed degradation when injecting DO292 Table E-8 DME/TACAN signals into a Rx simulator.

Then, we compare these results with the predicted degradation using proposed degradation formula (10).

\begin{tabular}{|l|l|l|l|l|l|}
\hline \multicolumn{4}{|l|}{ 20-MHz Filter } & 12-MHz Filter \\
\hline$B_{T H}=-120 \mathrm{dBW}$ & $\begin{array}{l}\text { DO292 Table } \\
10-3 \text { Results }\end{array}$ & $\begin{array}{l}\text { DO292 } \\
\text { Analytical } \\
\text { Results (8) }\end{array}$ & $\begin{array}{l}\text { Proposed } \\
\text { Method (10) }\end{array}$ & $\begin{array}{l}\text { DO292 Analytical } \\
\text { Results (8) }\end{array}$ & $\begin{array}{l}\text { Proposed Method } \\
(10)\end{array}$ \\
\hline $\mathrm{bdc}$ & 0.6121 & 0.6190 & 0.6190 & 0.4824 & 0.4824 \\
\hline \multicolumn{1}{|c|}{$R_{I}$} & 0.5424 & 0.5955 & 0.3617 & 0.6822 & 0.3778 \\
\hline C/N0 Degradation & 5.99 & 6.22 & 5.53 & 5.12 & 4.25 \\
\hline
\end{tabular}

As we can see, the result of the application of our formula (10) provides predicted C/N0 degradation values which are closer to the observed C/N0 degradation from our Rx simulator than to the results given in DO292 table 10-3.

Indeed, table 10-3 of DO-292 shows the predicted degradation results computed at the time with the same DME/TACAN database with DO292 degradation formula for BW=20 Mhz receiver. These results are recalled in column 2 of the above table. The predicted bdc is very similar, but the predicted degradation given in DO292 table 10-3 is equal to $5.99 \mathrm{~dB}$ and is larger than the observed degradation of $5.59 \mathrm{~dB}$ when inserting simulated DME/TACAN in our GNSS receiver simulator for BW=20 MHz.

Also, we see that re-computation of the $\mathrm{C} / \mathrm{N} 0$ degradation using the strict application of formula (8) from DO292 gives results which are strangely offset from DO292 table 10-3 and from our results using formula (10) or observed from Rx simulations.

We consider then that this DME/TACAN environment from DO292 table E-8 served to validate that our formula (10) better reflects reality and is then proposed to be used in the following calculations instead of DO292 formula (8).

\section{B) Simulation results at 2018 US Hot Spot}

Table 3 shows the predicted C/N0 degradation values using our formula (10) with entries from the 2018 US DME/TACAN database.

\begin{tabular}{|c|c|c|c|c|}
\hline \multirow{2}{*}{ DO292 blanking } & \multicolumn{2}{|c|}{$\mathrm{B}_{\mathrm{TH}}=-121 \mathrm{dBW}$} & \multicolumn{2}{c|}{$\mathrm{B}_{\mathrm{TH}}=-120 \mathrm{dBW}$} \\
\cline { 2 - 5 } & $20 \mathrm{MHz} \mathrm{Rx}$ & $12 \mathrm{MHz} \mathrm{Rx}$ & $20 \mathrm{MHz} \mathrm{Rx}$ & $12 \mathrm{MHz} \mathrm{Rx}$ \\
\hline bdc & 0.6237 & 0.4868 & 0.5949 & 0.4642 \\
\hline Estimated $\mathrm{R}_{\mathrm{I}}$ & 0.7007 & 0.7323 & 0.8872 & 0.9232 \\
\hline Degradation $(\mathrm{dB})$ & $\mathbf{5 . 8 8}$ & 4.56 & 6.00 & 4.75 \\
\hline
\end{tabular}

Table 3 : Predicted GNSS L5 C/N0 degradation result when inserting simulated DME/TACAN signals at 2018 US database FL400 US hot spot using our formula (10).

We can also run the prediction of the GNSS L5 C/N0 degradation using eq (10) for a GNSS L5 Rx with BW=20 Mhz and with Bth=$121 \mathrm{dBW}$ for an aircraft at FL400 at various positions over the US. 


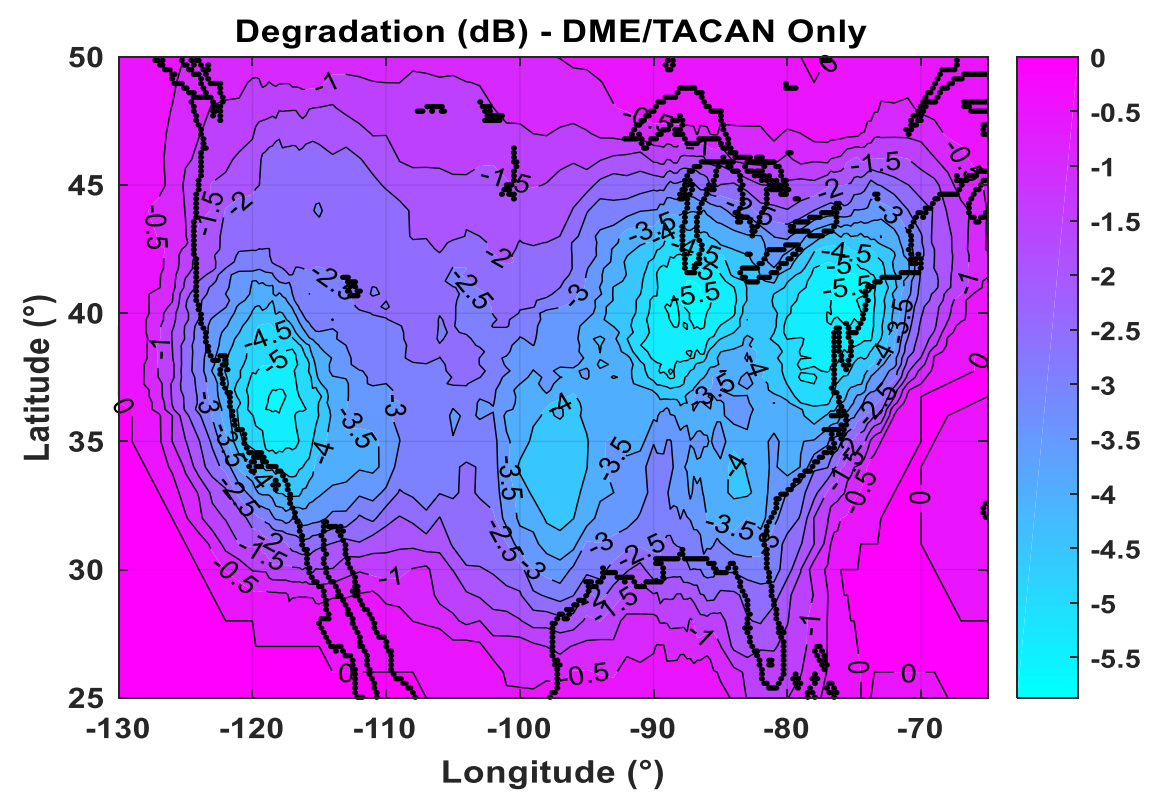

Figure 9 : Predicted GNSS L5 C/N0 degradation due to DME/TACAN using proposed eq (10) over the US at FL400 for a GNSS L5 Rx with BW=20 MHz and for Bth=-121 dBW.

The predicted largest value of the GNSS L5 C/NO degradation is $5.88 \mathrm{~dB}$ at FL400 for Rx BW=20 MHz and Bth=-121 dBW.

4.C) Simulation results at 2018 Europe Hot Spot

Table 4 shows the predicted C/N0 degradation values using our formula (10) with entries from the 2018 Europe DME/TACAN database.

\begin{tabular}{|c|c|c|c|c|}
\hline \multirow{2}{*}{ DO292 blanking } & \multicolumn{2}{|c|}{$\mathrm{B}_{\mathrm{TH}}=-121 \mathrm{dBW}$} & \multicolumn{2}{c|}{$\mathrm{B}_{\mathrm{TH}}=-120 \mathrm{dBW}$} \\
\cline { 2 - 5 } & $20 \mathrm{MHz} \mathrm{Rx}$ & $12 \mathrm{MHz} \mathrm{Rx}$ & $20 \mathrm{MHz} \mathrm{Rx}$ & $12 \mathrm{MHz} \mathrm{Rx}$ \\
\hline bdc & 0.5738 & 0.4066 & 0.5384 & 0.3687 \\
\hline Estimated $\mathrm{R}_{\mathrm{I}}$ & 0.5101 & 0.5306 & 0.6893 & 0.7139 \\
\hline Degradation $(\mathrm{dB})$ & $\mathbf{4 . 9 8}$ & 3.70 & 5.02 & 3.81 \\
\hline
\end{tabular}

Table 4 : Predicted GNSS L5 C/N0 degradation result when inserting simulated DME/TACAN signals at 2018 Europe database FL400 Europe hot spot using our formula (10).

The prediction of GNSS L5 C/N0 degradation over Europe using the 2018 database and using proposed eq (10) shows the predicted degradation map shown in the figure below. 


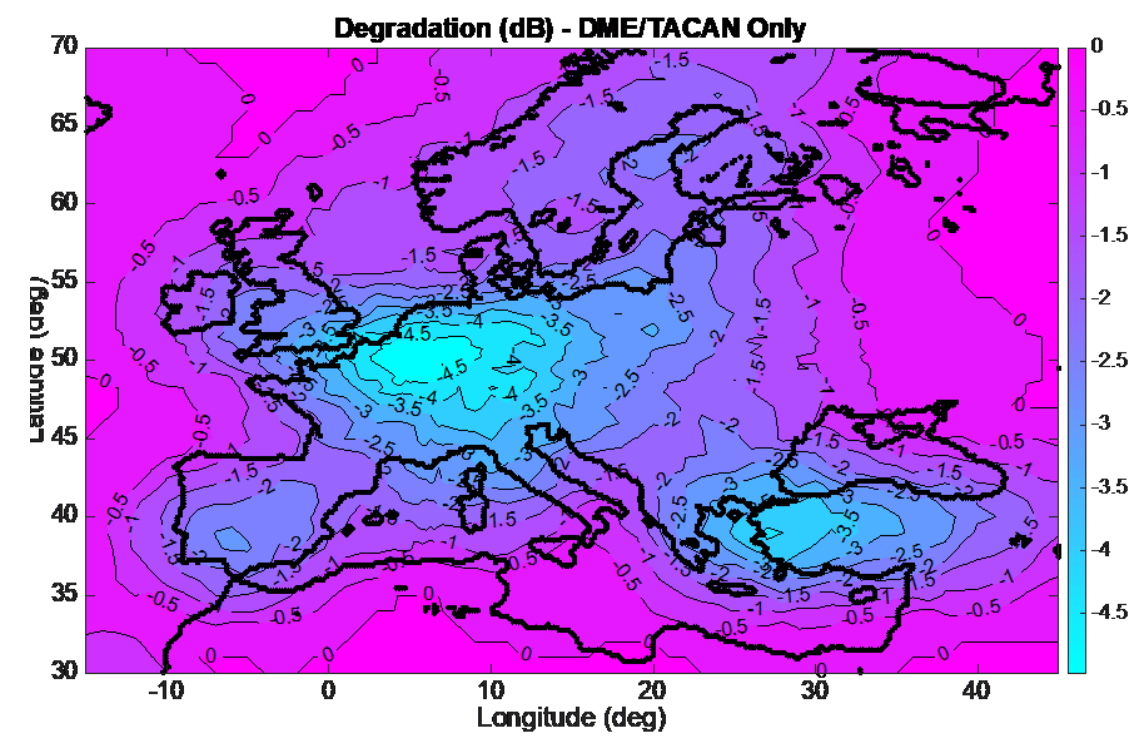

Figure 10 : Predicted GNSS L5 C/N0 degradation due to DME/TACAN using proposed eq (10) over Europe at FL400 for a GNSS L5 Rx with BW=20 MHz.

The predicted largest value of the GNSS L5 C/NO degradation is $4.98 \mathrm{~dB}$.

\section{CONCLUSION}

This paper has presented our proposed methodology to compute the GNSS L5 C/N0 degradation due to pulsed interference source presented in eq (10). The proposed formula is a refinement from the formula currently used in DO292 that should allow better fidelity of the prediction of $\mathrm{C} / \mathrm{N} 0$ degradation without too much pessimism.

The application of that formula requires the determination of the power spectral density of the pulsed interference source after the application of the blanker. In the case of DME/TACAN interference that post blanker power spectral density has been evaluated at US hot spot and it has been shown that this selected post blanker DME/TACAN psd is a satisfying overbound for the purpose of interference environment evaluation for standardization purposes.

That formula has then been applied and compared to existing DO292 results with the US DME/TACAN database of the time. Also, that formula has been run on the US and Europe 2018 DME/TACAN databases and the GNSS L5 C/N0 degradation maps have been drawn, to show a predicted largest value of the GNSS L5 C/NO degradation of $5.88 \mathrm{~dB}$ at FL400 for a Rx with BW=20 MHz and $\mathrm{Bth}=-121 \mathrm{dBW}$, while it is $4.98 \mathrm{~dB}$ over the Europe hot spot at FL 400 with an identical receiver.

\section{ACKNOWLEDGMENTS}

Dr. Olivier JULIEN's work contributing to this paper was exclusively performed when he was an ENAC employee.

\section{REFERENCES}

[Garcia- $\quad$ A.Garcia-Pena et al., "GNSS C/N0 Degradation Model in Presence of Continuous Wave and Pulsed Interference" Pena et al., Proceedings of the 2020 International Technical Meeting of The Institute of Navigation, Reston, San Diego, January 2020] 2020.

[Bastide, F. Bastide, Analysis of the Feasibility and Interests of Galileo E5a/E5b and GPS L5 for Use with Civil Aviation, 2004] Ph.D. thesis dissertation, INPT, 2004

[RTCA, RTCA, DO 292 - Assessment of RF interference relevant to the GNSS L5-E5a band, July 29, 2004

2004]

[Julien et Olivier Julien, Paul Verlaine Gakne, Axel Garcia-Pena, Christophe Macabiau, Mikael Mabilleau, Pierre Durel al., 2019] (2019), Efficient DME/TACAN Blanking Method for GNSS-based Navigation in Civil Aviation, ION GNSS+2019 
[Gao et al., Gao, G. X., Heng, L., Hornbostel, A., Denks, H., Meurer, M., Walter, T., \& Enge, P. (2013). "DME/TACAN 2013] Interference Mitigation for GNSS: Algorithms and Flight Test Results". GPS Solutions, 17(4), 561-573

[Grabowski Grabowski, J., \& Hegarty, C. (2002). "Characterization of L5 Receiver Performance Using Digital Pulse Blanking”. et al., 2002] Procedings of the Institute of Navigation GPS, (pp. 1630-1635). Portland, OR.

[Hegarty et Hegarty, C., Van Dierendonck, A. J., Bobyn, D., \& Grabowski, M. T. (2000). "Suppression of Pulsed Interference al., 2000] through Blanking". Proceedings of the IAIN World Congress and the 56th Annual Meeting of The Institute of Navigation , (pp. 399 - 408). San Diego, CA.

[Shallberg Shallberg, K., Flake, J., Baraban, D., \& Hegarty, C. (2018). "Updated Aviation Assessment of Interference in the et al., 2018] L5/E5A Bands from Distance Measuring Equipment". Proceedings of the 31st International Technical Meeting of The Satellite Division of the Institute of Navigation (ION GNSS+ 2018), (pp. 1324-1337). Miami, Florida.

[Raimondi, M. Raimondi, Development and Characterization of Pulsed Interference Mitigation Techniques for onboard GNSS 2008] Receivers, Ph.D. Thesis, INPT, 2008 